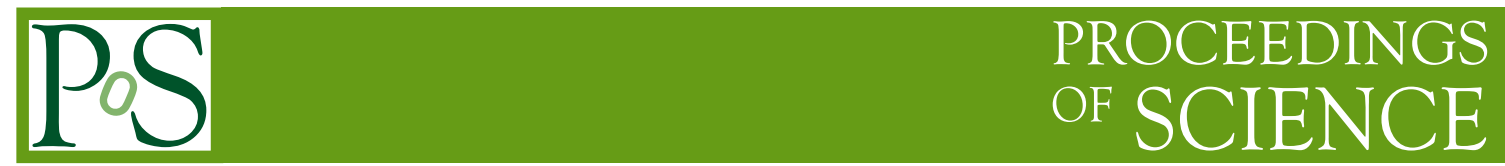

\title{
Soft QCD measurements with ALICE
}

\author{
Beomkyu Kim] for the ALICE Collaboration \\ Inha Univ. \\ E-mail: kimbecern.ch
}

Particle production at LHC energies involves perturbative (hard) and non-perturbative (soft) QCD regimes. At low transverse momentum it is generally described by phenomenological models. In this contribution, we report on soft QCD variables, such as inelastic, single- and double-diffractive cross sections, charged-particle multiplicity density and observables characterising the Underlying Event. Results are presented for data from pp, $\mathrm{p}-\mathrm{Pb}, \mathrm{Xe}-\mathrm{Xe}$ and $\mathrm{Pb}-\mathrm{Pb}$ collisions at $\mathrm{LHC}$ energies reconstructed with the ALICE detector. The evolution of particle production with energy and system size is discussed.

The 39th International Conference on High Energy Physics (ICHEP2018)

4-11 July, 2018

Seoul, Korea

${ }^{*}$ Speaker. 


\section{Introduction}

The measurements of inelastic and diffractive cross sections in high energy proton-proton (pp) collisions contribute to the theoretical understanding of QCD in the non-perturbative regime. The single- and double-diffractive processes (SD and DD) have a relevant contribution to the inelastic pp cross section $\left(\sigma_{\mathrm{INEL}} \sim \sigma_{\mathrm{SD}}+\sigma_{\mathrm{DD}}+\sigma_{\mathrm{ND}}\right)$. Particle production in high energy pp collisions can be described by considering a perturbative hard partonic scattering surrounded by the so-called Underlying Event (UE). The Underlying Event includes Initial and Final State Radiations (ISR+FRS), colour-connected beam remnants and infrared Multi-Parton Interactions (MPI). For central AA collisions at LHC energies, a new phase of matter, the so-called Quark-Gluon Plasma (QGP), is created and hadronises gradually to final-state particles with the transverse momentum of few $\mathrm{GeV} / c$. As a result, the Underlying Event resulting from the QGP is mainly soft, and the hard partonic scatterings occurring at early stages of AA collisions can be used as a probe of the QGP medium. Experiments at the LHC have reported the observation of QGP-like behaviours even in pp collisions [1, 2]. Measurements like charged-particle multiplicity density $\mathrm{d} N_{\mathrm{ch}} / \mathrm{d} \eta$ as a function of system size and energy provide valuable information to understand the origin of the similarities between the two collision systems. In this work we report recent results on soft QCD observables using data reconstructed with the ALICE detector.

\section{ALICE detectors}

The soft QCD observables are measured using the V0, ITS and TPC detectors of ALICE [3]. The SPD is a sub-detector of the ITS that measures short track segments (tracklets) in the pseudorapidity range $|\eta|<2$. The $\mathrm{V} 0$ detectors are two scintillator hodoscopes that are located on either side of the interaction point at $z=3.3 \mathrm{~m}$ and $-0.9 \mathrm{~m}$, respectively, along the beam direction. They cover the pseudorapidity region $2.8<\eta<5.1$ and $-3.7<\eta<-1.7$ in full azimuth and are employed for triggering, background suppression and event-class determination. The combined data of the ITS and TPC provide 4-momentum information of charged particles in the mid-rapidity $|\eta|<0.9$ at ALICE. Inelastic cross section is determined using data taken with a minimum-bias trigger, while the luminosity is measured with a van der Meer scan [4]. Single- and double-diffraction cross sections are measured using the additional rapidity windows of the above detectors [5]. The charged-particle multiplicity density is measured by counting the number of tracklets using the SPD. The Underlying Event as a function of the highest charged-particle transverse momentum $\left(p_{\mathrm{T}}^{\text {leading }}\right)$ is studied in terms of the number and summed $p_{\mathrm{T}}$ densities of charged particles in the azimuthal regions that are towards and transverse to the $p_{\mathrm{T}}^{\text {leading }}$ direction using the track information given by the ITS and TPC.

\section{Results and discussions}

Table 1 shows single-, double-diffractive and inelastic cross sections in pp collisions at different collision energies measured by ALICE [5]. The contribution from diffractive processes to the inelastic cross section $\left(\left(\sigma_{\mathrm{SD}}+\sigma_{\mathrm{DD}}\right) / \sigma_{\mathrm{INEL}}\right)$ is measured around $30 \%$ at LHC energies. Within uncertainties ALICE measurements are compatible with those from other experiments. It indicates 
Table 1: Diffractive and inelastic cross sections in pp collisions at ALICE [5].

\begin{tabular}{c|c|c|c}
\hline$\sqrt{s}(\mathrm{TeV})$ & $\sigma_{\mathrm{SD}}(\mathrm{mb})$ & $\sigma_{\mathrm{DD}}(\mathrm{mb})$ & $\sigma_{\mathrm{INEL}}(\mathrm{mb})$ \\
\hline 0.9 & $11.2_{-2.1}^{+1.6}($ syst $)$ & $5.6 \pm 2.0($ syst $)$ & \\
2.76 & $12.2_{-5.3}^{+3.9}($ syst $) \pm 0.2($ lumi $)$ & $7.8 \pm 3.2($ syst $) \pm 0.2($ lumi $)$ & $62.8_{-4.0}^{+2.4}($ syst $) \pm 1.2($ lumi $)$ \\
7 & $14.9_{-5.9}^{+3.4}($ syst $) \pm 0.5($ lumi $)$ & $9.0 \pm 2.6($ syst $) \pm 0.3($ lumi $)$ & $73.2_{-4.6}^{+2.0}($ syst $) \pm 2.6($ lumi $)$ \\
\hline
\end{tabular}

that $\sim 70 \%$ of inelastic pp collisions is associated to the hard scattering residing with the Underlying Events at LHC energies.

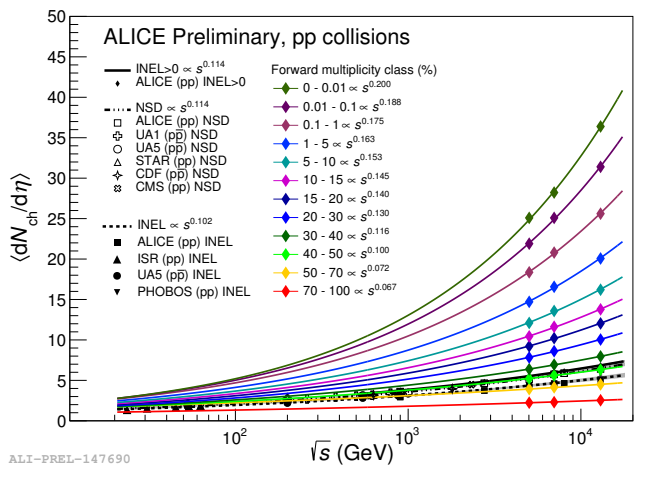

(a)

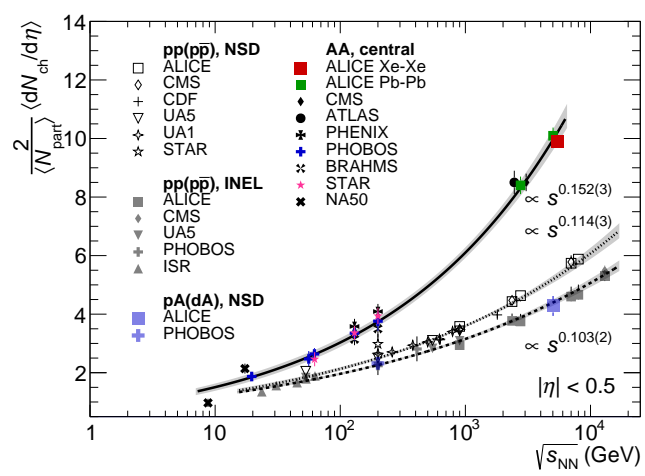

(b)

Figure 1: $\mathrm{d} N_{\mathrm{ch}} / \mathrm{d} \eta$ as a function of $\sqrt{s}$ for different multiplicity classes (a) and $\frac{2}{\left\langle N_{\mathrm{part}}\right\rangle}\left\langle\mathrm{d} N_{\mathrm{ch}} / \mathrm{d} \eta\right\rangle$ as a function of $\sqrt{s_{\mathrm{NN}}}$ for various collision systems (b).

Figure 1 a shows the mean charged-particle pseudorapidity density in $|\eta|<0.5$ as a function of $\sqrt{s}$ for different multiplicity classes in pp collisions. The multiplicity class is estimated using the energy amplitude of charged particles deposited in the V0 for a collision by comparing to the amplitude distribution of pp collisions. The $0-1 \%$ multiplicity class, for instance, indicates the top $1 \%$ high multiplicity pp collisions. For the same multiplicity class, $\mathrm{d} N_{\mathrm{ch}} / \mathrm{d} \eta$ are fitted with a power-law function. The fit shows a steeper particle production for a higher multiplicity class (smaller percentile) resulting from the MPI.

Figure $1 \mathrm{~b}$ shows $\frac{2}{\left\langle N_{\mathrm{part}}\right.}\left\langle\mathrm{d} N_{\mathrm{ch}} / \mathrm{d} \eta\right\rangle$ vs $\sqrt{s}, \sqrt{s_{\mathrm{NN}}}$ for various collision systems from pp to $\mathrm{Pb}-$ $\mathrm{Pb}$ and energies in the centre of mass from 0.9 to $13 \mathrm{TeV}$, where $\left\langle N_{\text {part }}\right\rangle$ is the average number of nucleons participating in a collision that is introduced to normalise and compare the yield of particle production in $\mathrm{pA}$ and AA to the case in pp collisions [6]. The $\mathrm{Xe}-\mathrm{Xe}(A=129)$ result is located in the trend made with heavy-ion results for the top 5\% central collisions indicating that $\mathrm{Pb}-\mathrm{Pb}$ and $\mathrm{Xe}-\mathrm{Xe}$ are consistent despite their substantial difference in mass. Also the $\mathrm{p}-\mathrm{Pb}$ results for Non-Single Diffractive (NSD = inelastic without SD) collisions are overlaid in the trend of pp inelastic collisions, which means that the yield coming from the diffractive processes is negligible in pA collisions. The particle yields as a function of $\sqrt{s}, \sqrt{s_{\mathrm{NN}}}$ at mid-rapidity are parameterised well by a power-law function up to LHC energies.

One observable sensitive to the Underlying Event in pp collisions is the summed $p_{\mathrm{T}}$ density as a function of $p_{\mathrm{T}}^{\text {leading }}$. Figure $2 \mathrm{a}$ shows results for the toward region in pp collisions at $\sqrt{s}=7$ and $13 \mathrm{TeV}$. This region mostly collects the fragmentation products of the hardest partonic interaction, which explains the monotonical increase of summed $p_{\mathrm{T}}$ with the $p_{\mathrm{T}}^{\text {leading }}$ scale. Figure $2 \mathrm{~b}$ shows the analogous result for the transverse region. Contrary to the toward side, a first steep rise of summed 


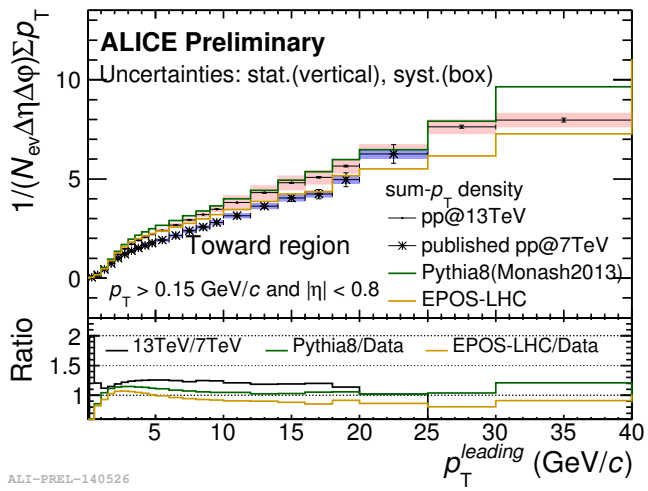

(a)

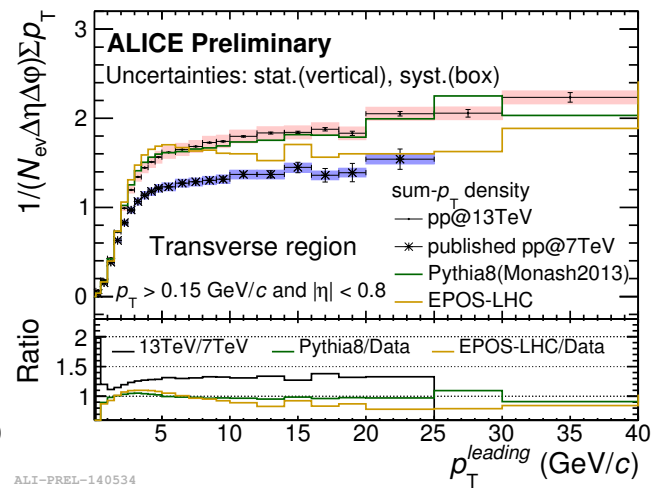

(b)

Figure 2: Sum- $p_{\mathrm{T}}$ densities as a function of $p_{\mathrm{T}}^{\text {leading }}$ in the toward (a) and transverse (b) regions.

$p_{\mathrm{T}}$ and then a saturation starting from $p_{\mathrm{T}}^{\text {leading }} \sim 5 \mathrm{GeV} / c$ are observed. It is worth noting that the activity in both toward and transverse sides as a function of $\sqrt{s}$ obeys scaling properties attributed to the energy dependence of soft particle production [7] in inelastic pp collisions.

\section{Conclusions}

The evolution of particle production with energy and system size that ALICE has measured for the last 8 years is summarised in this paper. About $70 \%$ of the inelastic cross section is due to hard partonic interactions, while the remaining $30 \%$ is associated to diffractive processes. The contribution from MPI is increased for high-multiplicity pp collisions and more study for additional constraints to the increased MPI can help to understand the QGP-like effects in small systems. The medium-sized $\mathrm{Xe}$-ion still behaves like the heavier $\mathrm{Pb}$-ion for the top 5\% central collisions in terms of particle production. The study of the Underlying Event can provide further understanding of the particle production mechanism as it is useful to distinguish soft and hard processes in pp collisions.

\section{References}

[1] G. Aad et al. [ATLAS Collaboration], Phys. Rev. Lett. 110 (2013) no.18, 182302 [arXiv:1212.5198 [hep-ex]].

[2] V. Khachatryan et al. [CMS Collaboration], Phys. Rev. Lett. 116 (2016) no.17, 172302 doi:10.1103/PhysRevLett.116.172302 [arXiv:1510.03068 [nucl-ex]].

[3] K. Aamodt et al. [ALICE Collaboration], JINST 3 (2008) S08002.

[4] S. van der Meer, CERN-ISR-PO-68-31.

[5] B. Abelev et al. [ALICE Collaboration], Eur. Phys. J. C 73 (2013) no.6, 2456 doi:10.1140/epjc/s10052013-2456-0 [arXiv:1208.4968 [hep-ex]].

[6] S. Acharya et al. [ALICE Collaboration], arXiv:1805.04432 [nucl-ex].

[7] A. Ortiz and L. Valencia Palomo, Phys. Rev. D 96 (2017) no.11, 114019 doi:10.1103/PhysRevD.96.114019 [arXiv:1710.04741 [hep-ex]]. 